\title{
Significance of Hyponatremia as an Independent Factor in Predicting Short- term Mortality in Patients with Hemorrhagic Stroke
}

\author{
Ali Shah ${ }^{1}$, Samurna Sabir ${ }^{2}$, Moiz Artani ${ }^{3}$, Osama Salam ${ }^{2}$, Shehroz Khan ${ }^{3}$, Amber Rizwan ${ }^{4}$ \\ 1. Internal Medicine, Jinnah Sindh Medical University, Karachi, PAK 2. Internal Medicine, Dow University \\ of Health Sciences, Karachi, PAK 3. Community Health Sciences, Jinnah Medical and Dental College, \\ Karachi, PAK 4. Family Medicine, Dr. Ruth Pfau Hospital, Karachi, PAK
}

$\square$ Corresponding author: Amber Rizwan, amber_aljazeera109@hotmail.com Disclosures can be found in Additional Information at the end of the article

\section{Abstract}

\section{Introduction}

Hyponatremia is the most common electrolyte imbalance in patients with acute cerebrovascular insults. In patients with acute non-traumatic hemorrhagic stroke, the role of hyponatremia as a negative prognostic indicator has been controversial. The aim of this study is to evaluate the frequency of hyponatremia in patients with hemorrhagic stroke and determine its impact on their in-hospital disease outcome.

\section{Methods}

This was a prospective observational study, which included all patients with non-traumatic hemorrhagic stroke. Serum sodium concentration $<135 \mathrm{mmol} / \mathrm{L}$ was labeled as hyponatremia. The etiology of hyponatremia was determined as syndrome of inappropriate antidiuretic hormone (SIADH) and cerebral salt wasting syndrome (CSWS). The outcome was categorized as "complete recovery," "motor/sensory deficit but not wheelchair/bed bound," "wheelchair/bed bound," and "in-hospital mortality.” SPSS for Windows version 22.0 (IBM Corp., Armonk, NY, US) was used to analyze the data.

\section{Results}

Out of 234 patients of hemorrhagic stroke, $45.3 \%(n=106)$ were hyponatremic, $58.5 \%$ had SIADH and $41.5 \%$ had CSWS. The overall mortality rate of hemorrhagic stroke was $16.2 \%$. The mortality rate was $36.5 \%$ in the SIADH group, $50 \%$ in the CSWS group, and $13.1 \%$ in the

Received 04/04/2019 Review began 04/08/2019 Review ended 04/14/2019 Published 04/27/2019

\section{(c) Copyright 2019}

Shah et al. This is an open access article distributed under the terms of the Creative Commons Attribution License CC-BY 3.0., which permits unrestricted use, distribution, and reproduction in any medium, provided the original author and source are credited. normonatremic group ( $\mathrm{p}<0.00001)$. The mean hospital stay in the SIADH group was $7.04 \pm 2.57$, in the CSWS group, it was $6.50 \pm 1.55$, and in the normonatremic group, it was $3.88 \pm 2.74$ $(\mathrm{p}=0.000)$.

\section{Conclusion}

Hyponatremia is an independent predictor of short-term mortality in patients with acute hemorrhagic stroke.

Categories: Family/General Practice, Internal Medicine, Neurology

Keywords: hyponatremia, mortality risk, in-hospital mortality, hemorrhagic stroke, predictors of

How to cite this article

Shah A, Sabir S, Artani M, et al. (April 27, 2019) Significance of Hyponatremia as an Independent Factor in Predicting Short-term Mortality in Patients with Hemorrhagic Stroke. Cureus 11(4): e4549. DOI 10.7759 /cureus. 4549 
mortality, disease outcome, stroke, prognosis, intracerebral hemorrhage

\section{Introduction}

Hyponatremia (serum sodium $<135 \mathrm{mmol} / \mathrm{l}$ ) is the most common electrolyte imbalance encountered not only in the general population but also in patients with acute ischemic and hemorrhagic stroke. Hyponatremia may be due to syndrome of inappropriate antidiuretic hormone (SIADH) or cerebral salt wasting syndrome (CSWS) [1-2].

Antidiuretic hormone (ADH) is a hormone of the pituitary gland that regulates water quantities in the body by acting on the kidneys. Physiologically, ADH is secreted in response to decreased plasma volume or increased serum osmolality. In SIADH, ADH is persistently secreted due to a failure of the negative feedback mechanism leading to increased water resorption from the kidneys and, consequently, dilutional hyponatremia [3]. On the other hand, hyponatremia in CSWS is due to large urinary losses of sodium [1].

Hyponatremia has been described as one of the risk factors predisposing to cerebrovascular stroke. Hyponatremia is also a complication of stroke. It has a crucial impact on the prognosis as well as the short-term and long-term mortality in patients with stroke [4]. In patients with hemorrhagic stroke (intracerebral hemorrhage (ICH)), hyponatremia was first evaluated by Kuramatsu et al. in 2014 [5]. They reported that in-hospital mortality was twice as common in hyponatremic patients as compared to normonatremic patients and the hyponatremia correction does not influence mortality [5]. This was a breakthrough publication which was succeeded by a few other research works, which reported higher mortality in hyponatremic patients of ICH [6-7].

Clinical data is still scarce. To the best of our knowledge, there exists only one study from Pakistan that evaluated the serum sodium levels in patients with ischemic stroke and found that $25 \%$ were mildly, $9.8 \%$ were moderately, and $3.8 \%$ were severely hyponatremic. They did not correlate serum sodium levels with either duration of hospital stay or short- or long-term mortality [8]. However, we could not come across any study predicting the association of hyponatremia with mortality in patients with ICH. The aim of this study is to evaluate the frequency of hyponatremia in patients with hemorrhagic stroke and determine their shortterm outcome.

\section{Materials And Methods}

This was a prospective observational study, which was conducted in the department of medicine in a tertiary care public hospital in Karachi over a period of six months (July-Dec 2018). The study was approved by the institutional review board, which waived patient consent. During the study period, there were 638 patients admitted with a clinical and neuroradiological diagnosis of stroke. Upon neuroimaging, there were 234 (36.6\%) established cases of hemorrhagic stroke that were included in the study and evaluated for hyponatremia. Serum sodium concentration $<135 \mathrm{mmol} / \mathrm{L}$ was labeled as hyponatremia [1]. Patients with a history of gastroenteritis, head trauma, brain tumor, pulmonary Kochs, bacterial pneumonia, bronchogenic carcinoma, leukemia, lymphoma, recent surgery, and patients who were taking any drugs that can cause hyponatremia were excluded from this study. Patients who died within the hospital emergency room and patients who died so early after admissions that their baselines labs weren't sent to or chased from the laboratory were also excluded, as even one serum sodium value was not available.

Hence, out of 234 cases of hemorrhagic stroke, 106 (45.3\%) hyponatremic patients aligned with the inclusion criteria and were included. When the patients were categorized according to the etiology of hyponatremia, CSWS was identified in 44 (41.5\%) patients, and SIADH was 


\section{Cureus}

identified in 62 (58.5\%) patients. Extracellular fluid volume, hematocrit, serum electrolytes, serum albumin, serum uric acid, and renal functions tests were included for all patients. CSWS and SIADH were evaluated as given in Table 1, which is adapted from Saleem et al. [1].

\begin{tabular}{lll} 
Clinical Parameters & CSWS & SIADH \\
\hline Hematocrit & Increased & Normal \\
Serum albumin concentration & Increased & Normal \\
BUN / creatinine & Increased & Decreased \\
Serum potassium & Normal or increased & Normal \\
Serum uric acid & Normal or increased & Decreased \\
Treatment & Normal saline & Fluid restriction
\end{tabular}

\section{TABLE 1: Clinical parameters for the diagnosis of CSWS and SIADH}

BUN: Blood urea nitrogen; SIADH: Syndrome of Inappropriate secretion of antidiuretic hormone; CSWS: Cerebral salt wasting syndrome

The site of hemorrhage was determined on magnetic resonance imaging (MRI) or magnetic resonance angiography (MRA). Non-contrast computed tomography (NCCT) was done for patients who were unable to tolerate MRI due to claustrophobia or had contraindications to it, including aneurysm clips, pacemakers, rods and screws, or any other magnetic materials in their bodies, or were on ventilatory life support as NCCT becomes more practical in these patients. Hemorrhagic sites identified for this study included left and right putamen, thalamic, and cerebellar hemorrhage and pontine hemorrhage. Patients were followed throughout their hospital stay to evaluate their duration of stay and outcome of stroke. The outcome was categorized as "complete recovery" when there was no deficit on examination; "motor/sensory deficit but not wheelchair/bed bound" when there was minor deficit upon discharge although the patient was not dependent; "wheelchair/bed bound" when the deficit was severe enough to render the patient wheelchair-bound or bedridden; and "in-hospital mortality" when the patient died after 24 hours of hospital admission. SPSS for Windows version 22.0 (IBM Corp., Armonk, NY, USA) was used to analyze the data. Frequencies and percentages were calculated for categorical variables and mean and standard deviation (SD) were calculated for continuous variables. Serum sodium status of the patients was compared to the hemorrhage site and inhospital outcome to deduce any relationships. Statistical significance for these relationships was calculated using Chi-square. P-value of $\leqslant 0.05$ was taken as significant.

\section{Results}

The incidence of hyponatremia in patients with hemorrhagic stroke was $45.3 \%(n=106)$. Out of these 106 patients, there were 83 (78.3\%) men and 23 (21.7\%) women. The male to female ratio was 3.5:1. Their mean age was $59.14 \pm 9.05$ years with men being slightly older (mean age: 60.55 \pm 4.81 years) than women (mean age: $59.07 \pm 1.11$ years). There were 13 (12.3\%) patients who have had a previous episode of stroke.

Patients were then categorized according to their site of hemorrhage. Overall, left putamen hemorrhage was the most common $(n=56 ; 52.8 \%)$ and pontine hemorrhage was the least 


\section{Cureus}

common ( $n=8 ; 7.5 \%)$. Among the patients with hyponatremia due to SIADH, right putamen hemorrhage was the most common $(n=14 ; 22.5 \%)$, followed by left putamen $(n=13 ; 20.9 \%)$ and left thalamic hemorrhage $(n=11 ; 17.7 \%)$. Among the patients with hyponatremia due to SIADH, left putamen hemorrhage was the most common $(n=11 ; 25 \%)$, followed by right putamen $(n=9$; $20.4 \%)$ and right thalamic hemorrhage $(n=8 ; 18.2 \%)$. Left putamen hemorrhage was also the most common site in patients who did not develop hyponatremia $(n=32 ; 25 \%)$ followed by right thalamic hemorrhage $(n=27 ; 21 \%)$. There was no statistical significance in the distribution of the hemorrhage site as compared to the status of serum sodium as shown in Table 2.

\begin{tabular}{|c|c|c|c|c|}
\hline $\begin{array}{l}\text { Site of } \\
\text { hemorrhagic } \\
\text { stroke }\end{array}$ & $\begin{array}{l}\text { Hyponatremia due to } \\
\text { SIADH }(n=62)\end{array}$ & $\begin{array}{l}\text { Hyponatremia due to } \\
\text { Csws }(n=44)\end{array}$ & $\begin{array}{l}\text { Patients with no } \\
\text { hyponatremia }(n=128)\end{array}$ & $\begin{array}{l}P \\
\text { value }\end{array}$ \\
\hline $\begin{array}{l}\text { Right putamen } \\
\text { hemorrhage }\end{array}$ & 14 (22.5\%) & $9(20.4 \%)$ & $23(17.9 \%)$ & 0.74 \\
\hline $\begin{array}{l}\text { Left putamen } \\
\text { hemorrhage }\end{array}$ & $13(20.9 \%)$ & $11(25 \%)$ & $32(25 \%)$ & 0.81 \\
\hline $\begin{array}{l}\text { Right thalamic } \\
\text { hemorrhage }\end{array}$ & 10 (16.2\%) & $8(18.2 \%)$ & 27 (21.0\%) & 0.70 \\
\hline $\begin{array}{l}\text { Left thalamic } \\
\text { hemorrhage }\end{array}$ & $11(17.7 \%)$ & 7 (15.9\%) & 14 (10.9\%) & 0.39 \\
\hline $\begin{array}{l}\text { Right cerebellar } \\
\text { hemorrhage }\end{array}$ & 9 (14.5\%) & $5(11.3 \%)$ & 16 (12.5\%) & 0.88 \\
\hline $\begin{array}{l}\text { Left cerebellar } \\
\text { hemorrhage }\end{array}$ & $3(4.8 \%)$ & $1(2.2 \%)$ & 13 (10.2\%) & 0.15 \\
\hline Pontine hemorrhage & $2(3.2 \%)$ & 3 (6.8\%) & 3 (2.3\%) & 0.36 \\
\hline
\end{tabular}

\section{TABLE 2: Distribution of hemorrhagic site according to the frequency of hyponatremia due to SIADH and CSWS and patients with no hyponatremia}

SIADH: Syndrome of Inappropriate secretion of antidiuretic hormone; CSWS: Cerebral salt wasting syndrome

The outcome of stroke was categorized on four levels and compared to the serum sodium status. The mortality rate of hemorrhagic stroke was $16.2 \%$; $50 \%$ of the deceased patients had hyponatremia due to CSWS. There were $20.9 \%$ who were rendered wheelchair or bed-bound; $38.7 \%$ of these did not develop hyponatremia. There were $42.7 \%$ of patients who had a motor or sensory deficit but not wheelchair or bed bound; $73 \%$ of these did not develop hyponatremia; and $20 \%$ had hyponatremia due to SIADH. Only $20.1 \%$ of the patients recovered completely; $66 \%$ of these did not develop hyponatremia, $27.6 \%$ had hyponatremia due to SIADH, and 6.3\% had hyponatremia due to CSWS. The comparisons of the mean duration of hospital stay and the outcomes of stroke among the three groups of patients are shown in Table 3. 


\section{Cureus}

\begin{tabular}{|c|c|c|c|c|}
\hline Outcome of Stroke $(\mathrm{N}=234)$ & $\begin{array}{l}\text { Hyponatremia } \\
\text { due to SIADH }\end{array}$ & $\begin{array}{l}\text { Hyponatremia } \\
\text { due to Csws }\end{array}$ & $\begin{array}{l}\text { Patients with no } \\
\text { hyponatremia }\end{array}$ & P value \\
\hline Mean duration of hospital stay in days & $7.04 \pm 2.57$ & $6.50 \pm 1.55$ & $3.88 \pm 2.74$ & 0.000 \\
\hline Complete recovery $(n=47 ; 20.1 \%)$ & $13(27.6 \%)$ & $3(6.3 \%)$ & $31(66 \%)$ & 0.04 \\
\hline $\begin{array}{l}\text { Motor / sensory deficit but not } \\
\text { wheelchair/bed bound ( } n=100 ; 42.7 \%)\end{array}$ & $20(20 \%)$ & $7(7 \%)$ & $73(73 \%)$ & $<0.00001$ \\
\hline Wheelchair/bed bound ( $\mathrm{n}=49 ; 20.9 \%)$ & $15(30.6 \%)$ & $15(30.6 \%)$ & $19(38.7 \%)$ & 0.01 \\
\hline In-hospital mortality $(\mathrm{n}=38 ; 16.2 \%)$ & $14(36.8 \%)$ & $19(50 \%)$ & $5(13.1 \%)$ & $<0.00001$ \\
\hline
\end{tabular}

\section{TABLE 3: Outcome of stroke in patients with hyponatremia due to SIADH and CSWS and patients with no hyponatremia}

SIADH: Syndrome of Inappropriate secretion of antidiuretic hormone; CSWS: Cerebral salt wasting syndrome

\section{Discussion}

One of the most common acute electrolyte imbalances in patients with stroke is hyponatremia. It mimics the signs of underlying neurological deficit and worsens the disease outcome [1-2,5]. This study evaluated that almost half of the patients with hemorrhagic stroke developed hyponatremia. SIADH was a more common entity. The duration of hospital stay was significantly longer in patients with hyponatremia. Not only was the mortality rate higher in these patients, but the outcome of surviving patients, at the time of discharge, was worse in patients with hyponatremia.

This study is crucial is establishing hyponatremia as a negative prognostic factor in patients with non-traumatic hemorrhagic stroke. However, it has its limitations. The study followed the patients till discharge and has only reported short-term outcomes in these patients. It was a single-center, time-bound study, which was limited by the number of patients available for analysis. To establish hyponatremia as a negative prognostic factor and predictor of mortality, patients should have been followed for a longer term. Furthermore, not all patients had central venous pressures (CVP) measured, hence, the diagnosis of SIADH vs. CSWS might not be absolute. When CVP was not available, we used the fluid balance to distinguish SIADH from CSWS. Hence, our results are more clinical and based on real-world practices.

Hyponatremia was a previously unrecognized predictor of hospital stay and in-hospital mortality in patients with hemorrhagic stroke and was first studied in 2014 [5]. Since then, hyponatremia has been gaining wide recognition as a negative prognosis in cerebrovascular accidents. The frequency of hyponatremia has been reported from $12 \%-52 \%$ of patients with ischemic or hemorrhagic stroke. The frequency of hyponatremia in this is comparable to the literature [1-2,4-9]. We had a higher incidence of SIADH (58.5\%) as compared to CSWS (41.5\%).

The first time hyponatremia was established as a predictor of mortality in patients with hemorrhagic stroke, these patients were seen to have twice the mortality rate as compared to normonatremic patients [5]. The second study done to reinforce this association reported a higher mortality rate in normonatremic patients as compared to hyponatremic (32\% vs. $24 \%$ ), the difference was not statistically significant. However, hyponatremic patients had a 
significantly longer median duration of hospital stay [7]. Carcel et al. then studied the effect of hyponatremia in the outcome of hemorrhagic stroke in a large sample. At 90 days, the mortality rate in the hyponatremic group was $18 \%$ and in the normonatremic group was $11 \%$.

Hyponatremic patients had a 1.8 times higher risk of death as compared to the normonatremic patients $(\mathrm{p}<0.001)$. However, their hyponatremic patients did not have a statistically significant risk of major disability after multivariable adjustment [6]. However, Kalita et al. failed to show hyponatremia as a negative prognostic factor of stroke. In their study, death was higher in hypernatremic patients as compared to hyponatremic and normonatremic patients $(p=.04)$ [9].

Whether or not hyponatremia can be established as a negative indicator of short- and longterm prognosis of hemorrhagic stroke is still not established through literature. Some literature does report otherwise; however, most studies have proposed the association. This literature helps neurologists and medical specialists to identify high-risk patients earlier, at the time of admission. Periodic serum sodium monitoring and in-time interventions can help prevent adverse outcomes in these patients.

\section{Conclusions}

Hyponatremia is a very common electrolyte imbalance reported in patients with acute cerebrovascular insult. The clinical significance of hyponatremia was undermined previously. However, current research has helped in establishing the crucial role of serum sodium levels in predicting short-term outcomes in patients with acute hemorrhagic stroke. Close monitoring of serum sodium levels in these patients, determination of the etiology of hyponatremia, and appropriate medical intervention can improve the outcome in these patients.

\section{Additional Information}

\section{Disclosures}

Human subjects: Consent was obtained by all participants in this study. Institutional Review Board, Civil Hospital issued approval CH/IRC/18/057. Animal subjects: All authors have confirmed that this study did not involve animal subjects or tissue. Conflicts of interest: In compliance with the ICMJE uniform disclosure form, all authors declare the following: Payment/services info: All authors have declared that no financial support was received from any organization for the submitted work. Financial relationships: All authors have declared that they have no financial relationships at present or within the previous three years with any organizations that might have an interest in the submitted work. Other relationships: All authors have declared that there are no other relationships or activities that could appear to have influenced the submitted work.

\section{References}

1. Saleem S, Yousuf I, Gul A, Gupta S, Verma S: Hyponatremia in stroke. Ann Ind Acad Neurol. 2014, 17:55-57. 10.4103/0972-2327.128554

2. Soiza RL, Cumming K, Clark AB, et al.: Hyponatremia predicts mortality after stroke. Int J Stroke. 2015, 10:50-55. 10.1111/ijs.12564

3. Gross P: Clinical management of SIADH. Ther Adv Endocrinol Metab. 2012, 3:61-73.

4. Rodrigues B, Staff I, Fortunato G, McCullough LD: Hyponatremia in the prognosis of acute ischemic stroke. J Stroke Cerebrovasc Dis. 2014, 23:850-854.

10.1016/j.jstrokecerebrovasdis.2013.07.011

5. Kuramatsu JB, Bobinger T, Volbers B, et al.: Hyponatremia is an independent predictor of inhospital mortality in spontaneous intracerebral hemorrhage. Stroke. 2014, 45:1285-1291. 10.1161/STROKEAHA.113.004136

6. Carcel C, Sato S, Zheng D, et al.: Prognostic significance of hyponatremia in acute intracerebral hemorrhage: pooled analysis of the intensive blood pressure reduction in acute cerebral hemorrhage trial studies. Critical Care Med. 2016, 44:1388-1394. 


\section{Cureus}

7. Gray J, Morbitzer K, Liu-DeRyke X, Parker D, Zimmerman L, Rhoney D: Hyponatremia in patients with spontaneous intracerebral hemorrhage. J Clin Med. 2014, 3:1322-1332.

10.3390/jcm3041322

8. Ahmed MS, Memon SF, Mustafa S, Javed A, Butt SM: Evaluation of hyponatremia in ischemic stroke patients in a tertiary care hospital of Karachi, Pakistan. Cureus. 2019, 11:3926. 10.7759/cureus.3926

9. Kalita J, Singh RK, Misra UK: Cerebral salt wasting is the most common cause of hyponatremia in stroke. J Stroke Cerebrovasc Dis. 2017, 26:1026-1032.

10.1016/j.jstrokecerebrovasdis.2016.12.011 